\title{
Reporte de caso clínico \\ Primer diagnóstico de Rhodococcus equi confirmado, laboratorialmente, en un potrillo de Costa Rica
}

\section{Clinical case report: First laboratory-confirmed diagnosis of Rhodococcus equi in a foal in Costa Rica}

\section{Relato de caso clínico: Primeiro diagnóstico de Rhodococcus equi confirmado, laboratorial- mente, em um potro em Costa Rica}

Roberto Estrada ${ }^{1}$, Carla Murillo ${ }^{1}$, Patricio Razquin ${ }^{1}$, Elías Barquero-Calvo ${ }^{2}$, Carlos Chacón-Díaz ${ }^{3}$, Manuel Estrada ${ }^{1 \otimes}$

1 Hospital Equino, Escuela de Medicina Veterinaria, Universidad Nacional, Costa Rica, roberto.estrada.mcdermott@una. cr, carla.murillo.ramirez@una.cr, patorazquin@gmail.com, juan.estrada.umana@una.cr

2 Programa de Investigación en Enfermedades Tropicales, Escuela Medicina Veterinaria, Universidad Nacional, Costa Rica, elias.barquero.calvo@una.cr

3 Centro de Investigación en Enfermedades Tropicales, Facultad Microbiología, Universidad de Costa Rica, Costa Rica, carlos.chacondiaz@ucr.ac.cr

Recibido: 21 de octubre de 2019 Corregido: 6 de marzo de 2020 Aceptado: 9 de marzo de 2020

\section{Resumen}

Neumonía por Rhodococcus equi es importante causa de morbilidad y mortalidad en potrillos de todo el mundo. La prevalencia y/o virulencia de la bacteria es, aparentemente, baja en Costa Rica, pues nunca han sido reportados brotes. Los casos sospechosos muestran solamente signos clínicos de leves a moderados. Sin embargo, un diagnóstico definitivo de la enfermedad nunca ha sido hecho anteriormente en caballos costarricenses. $R$. equi es una bacteria $\mathrm{G}(+)$ facultativa intracelular que causa neumonía en potrillos jóvenes (hasta 4-5 meses de edad). Este caso corresponde a un potrillo Frisón de cuatro semanas de edad que fue referido para cirugía al Hospital Equino, Universidad Nacional de Costa Rica, con una fractura de húmero y onfaloflebitis. En el período postoperatorio, el paciente presentó: signos de secreción nasal bilateral moderada, tos, fiebre, linfocitosis y neutrofilia. La evaluación radiológica del tórax mostró un patrón bronquialveolar en el aspecto ventrocraneal de los pulmones. La citología del aspirado transtraqueal confirmó un cuadro de bronconeumonía supurativa. Las muestras fueron cultivadas aeróbicamente en agar Columbia $+5 \%$ sangre a $37^{\circ} \mathrm{C}$ por $24 \mathrm{~h}$. Las características macroscópicas, microscópicas, bioquímicas y la identificación, por medio de la secuenciación parcial del gen "16SrRNA", de la bacteria aislada fueron compatibles con R. equi. El potrillo fue eutanasiado debido a re-fractura del húmero antes de que el diagnóstico de $R$. equi fuera confirmado. $R$. equi tiene el potencial de causar un severo impacto monetario a la industria de cría equina, por lo cual, es

\ Correo de correspondencia: juan.estrada.umana@una.cr 
importante hacer el diagnóstico y tratamiento precisos para evitar el surgimiento de cepas resistentes. Son necesarios más estudios para determinar la prevalencia local del patógeno y evaluar su impacto.

Palabras Clave: Rhodococcus equi, 16SrRNA, bronconeumonía potrillos, impacto monetario, Costa Rica

\begin{abstract}
Rhodococcus equi pneumonia is an important cause of morbidity and mortality in foals all over the world. Prevalence and/or virulence of bacteria is apparently low in Costa Rica since no outbreaks have been reported and suspicious cases show only mild to moderate clinical signs. However, there has never been a definitive diagnosis of the disease in Costa Rican horses. $R$. equi is a G (+) facultative intracellular bacteria causing pneumonia in young foals (up to 4-5 months of age). This paper refers to a 4-week old Friesian foal with a humerus fracture and omphalophlebitis that was referred to the Equine Hospital, National University, Costa Rica, for surgery. In the postoperatory period, the patient showed signs of moderate bilateral nasal discharge, cough, fever, lymphocytosis, and neutrophilia. The radiological evaluation of the thorax showed a bronchoalveolar pattern in the ventro cranial region of the lungs. The transtracheal aspirate cytology confirmed the presence of suppurative bronchopneumonia. The samples were aerobically cultured in Columbia agar $+5 \%$ blood at $37^{\circ} \mathrm{C}$ for $24 \mathrm{~h}$. The macroscopic, microscopic, and biochemical characteristics of the isolated bacteria, in addition to the partial sequencing of the "16SrRNA" gene, were compatible with $R$. equi. The foal was euthanized due to the humerus re-fracture before the R. equi diagnosis was confirmed. $R$. equi can potentially cause a severe monetary impact in the equine breeding industry; consequently, accurate diagnosis and treatments are important to avoid the emergence of resistant strains. Further studies are needed to determine the local prevalence of the pathogen and to evaluate its impact.
\end{abstract}

Keywords: Rhodococcus equi, 16SrRNA, foal bronchopneumonia, monetary impact, Costa Rica

\begin{abstract}
Resumo
A pneumonia por Rhodococcus equi é uma das principais causas de morbidade e mortalidade em potros em todo o mundo. A prevalência e / ou virulência da bactéria é, aparentemente, baixa em Costa Rica, pois nunca tem sido relatados surtos. Casos suspeitos mostram apenas sinais clínicos leves a moderados. No entanto, nunca tem sido feito um diagnóstico definitivo da doença em cavalos da Costa Rica. R. equi é uma bactéria G (+)facultativa intracelular que causa pneumonia em potros jovens (até 4-5 meses de idade). Esse caso corresponde a um potro Frisón de quatro semanas de idade que foi encaminhado para cirurgia no Hospital de Equinos, Universidade Nacional da Costa Rica, com uma fratura de úmero e onfaloflebite. No pós-operatório, o paciente apresentou sinais de secreção nasal bilateral moderada, tosse, febre, linfocitose e neutrofilia. A avaliação radiológica do tórax mostrou padrão bronquialveolar no aspecto ventrocraniano dos pulmões. A citologia do aspirado transtraqueal confirmou um quadro de broncopneumonia supurativa. As amostras foram cultivadas aerobicamente em ágar Columbia $+5 \%$ sangue a $37^{\circ} \mathrm{C}$ por $24 \mathrm{~h}$. As características macroscópicas, microscópicas, bioquímicas e a identificação, por meio do seqüenciamento parcial do gene "16SrRNA", da bactéria isolada foram compatíveis com R. equi. O potro foi eutanasiado devido à re-fratura do úmero antes de que o diagnóstico de $R$. equi fosse confirmado. $R$. equi tem o potencial de causar um severo impacto monetário para a indústria de cria de equinos, portanto, é importante fazer o diagnóstico e o tratamento precisos para evitar o surgimento de cepas resistentes. Mais estudos são necessários para determinar a prevalência local do patógeno e avaliar seu impacto.
\end{abstract}

Palavras-chave: Rhodococcus equi, 16SrRNA, broncopneumonia do potro, impacto monetário, Costa Rica

\title{
Introducción
}

Rhodococcus equi es una importante causa de morbilidad y mortalidad en potrillos de todo el mundo (Zachary 2017). Un diagnóstico definitivo de esta enfermedad, en caballos, no se ha hecho antes en 
Rev. Ciencias Veterinarias, Vol. 38, N 1, [43-52], E-ISSN: 2215-4507, enero-junio, 2020

DOI: https://doi.org/10.15359/rcv.38-1.4

URL: http://www.revistas.una.ac.cr/index.php/veterinaria/index

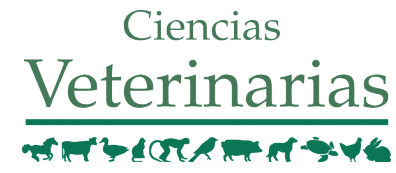

este país por lo cual, se considera de importancia el reporte de este caso clínico. Los Rhodococcus son bacilos Gram positivos, no móviles y aerobios, del orden Actinomycetales. Aparecen como filamentos que se fragmentan al separarse en bacilos y cocos (Prescott et al. 1997; Forbes et al. 1998; Guiguère 2010; Markley et al. 2013). Rhodococcus equi es un patógeno intracelular, facultativo, (Kelly et al. 2002) que puede vivir y reproducirse en macrófagos, donde resiste fagocitosis, gracias a que tiene varios factores de virulencia tales como: la proteína VapA (del Inglés: Virulence Associated Protein A); resistencia a los antibióticos betalactámicos; presencia de ácidos micólicos y cadenas largas de carbono en su pared celular, importante en formación de granulomas (Guiguère 2010); producción de colesterol oxidasa (Letek et al. 2008) y formación de biopelículas (Al Akhrass et al. 2012; Gressler et al. 2015). Presenta, además, un plásmido de virulencia (Takai et al. 2000; Letek et al. 2008), que acarrea genes (ej. resistencia antibiótica) los cuales benefician la supervivencia de la bacteria (Giguère et al. 2010), el proceso infeccioso y la proliferación en macrófagos.

\section{Epidemiología y Patogénesis}

Prescott (1987) publica reportes sobre la epidemiología de esta infección en caballos. $R$. equi habita suelos superficiales $(<30 \mathrm{~cm}$ profundidad), secos y polvorientos, afectando principalmente caballos y cabras (Muscatello et al. 2007). Es posible aislar la bacteria a partir de heces de la mayoría de los caballos adultos. Un estudio, efectuado con 117 yeguas en Kentucky, USA, concluyó que "detectar la bacteria en heces de yeguas sugiere que estas pueden ser importante fuente ambiental de R. equi" (Grimm et al. 2007). Cohen et al. (2008) determinaron que " $R$. equi fue comúnmente aislado del suelo en fincas de cría caballar", así como en heces de potrillos desde 1 semana de edad. La bacteria no se multiplica en el ambiente anaerobio de intestino grueso adulto, pero sí lo hace en el intestino del potrillo, hasta los tres meses de edad. Cuando el potrillo neumónico deglute esputo contaminado con $R$. equi, este se multiplica en su intestino, alcanza grandes concentraciones, razón por la cual los excrementos de potrillos infectados son fuente importante de contaminación ambiental con cepas virulentas. Bajo condiciones climáticas de temperatura alta, la bacteria se multiplica hasta por 10.000 veces en 2 semanas, de forma que un gramo de suelo contaminado puede llegar a contener millones de organismos de $R$. equi virulentos. En fincas con problemas endémicos, la bacteria se cultiva con mayor facilidad en el aire de establos que en los potreros (Kuskie et al. 2011; Cohen et al.2012; Cohen et al. 2013), particularmente, en días secos y ventosos. Así, los potrillos se exponen más a R. equi virulento cuando están estabulados. La inhalación del patógeno es la principal ruta para infección pulmonar (Cohen et al. 2012; Cohen et al. 2013). El tiempo de incubación oscila entre 8 y 15 días. Datos epidemiológicos sugieren que la mayoría de los potrillos se infecta como neonatos, pues el diagnóstico se hace, generalmente, antes de cumplir 40 días, aunque ocurren casos en edades más avanzadas (3-4 meses), al presentar importantes tasas de mortalidad (Losinger et al. 2000). La incidencia de neonatos afectados puede esperarse que sea mayor en fincas con gran densidad de yeguas y potrillos (Cohen et al. 2008). "Aunque R. equi se cultiva a partir de muestras ambientales, en la mayoría de las fincas de cría equina, la enfermedad clínica de potrillos puede ser endémica y devastadora en algunas yeguadas, esporádica en otras y no reconocida en la mayoría" (Guiguère 2010). La tasa de mortalidad oscila entre 30-40 \% de los potrillos infectados. Además, de neumonía insidiosa, granulomatosa, severa y progresiva, con abscesos de pulmón, esta bacteria puede afectar otras estructuras del cuerpo, tales como vértebras y otros huesos, linfonodos 
abdominales, ojos, articulaciones y cerebro. También, afecta vacas, perros, gatos y seres humanos (Lasky et al. 1991; Al Akhrass et al. 2012), especialmente pacientes inmunocomprometidos, al causar sintomatología clínica similar a la tuberculosis. En Costa Rica, el primer aislamiento se realizó en una niña de 2 días de edad (Herrera et al. 2000). También, la prevalencia y virulencia de R. equi, en la especie equina, aparentemente es baja pues nunca han sido reportados brotes; los casos sospechosos muestran solo signos moderados. Otra especie, en la cual se aisló R. equi (Álvarez et al. 2001) incluye las dantas (Tapirus bairdii). La combinación de un macrólido (eritromicina, azitromicina o claritromicina) con rifampicina es todavía un protocolo recomendado para potrillos con signos clínicos de infección por R. equi; sin embargo, la resistencia a estos antibióticos está aumentando (Giguère et al. 2010); por lo cual, se debe considerar otros tratamientos. La mayoría de los pacientes, con lesiones pulmonares subclínicas y ultrasonográficamente pequeñas, se recuperan sin terapia (Giguère 2017).

\section{Vacunación}

Investigadores han desarrollado una vacuna que protege a los potrillos contra infección por $R$. equi, pues elige, como blanco, un antígeno de superficie denominado poly-N-acetyl glucosamina (PNAG) (Cywes-Bentley et al. 2018), el cual se encuentra en muchos microbios. Lo anterior, implica que dicha vacuna tiene el potencial de proteger, también, contra otras enfermedades bacterianas, pero todavía no ha sido lanzada al mercado. Hasta agosto del 2019, no se consigue, a nivel mundial, una vacuna comercial autorizada para inmunizar potrillos contra neumonía por R. equi.

\section{Materiales y Métodos}

\section{Anamnesis}

Un potrillo Frisón, a los 15 días de edad, presentó una onfaloflebitis con absceso local, de contenido purulento caseoso; el cual se drenó, quirúrgicamente, en la finca de origen. La muestra de pus, tomada en este momento por hisopado, no cultivó nada. El dueño lo trató con estreptomicina/penicilina i.m. por 4 días y ocurrió regresión parcial de la inflamación umbilical. Dos semanas después, sufrió una fractura expuesta de radio, por lo cual, se refirió al Hospital Equino, Escuela de Medicina Veterinaria, Universidad Nacional, Costa Rica. Aquí fue operado, bajo anestesia general inhalatoria, para implantarle la placa y tornillos del caso. Se trató, profilácticamente, con Ceftiofur i.m. y Meglumina de Flunixin i.v. por 5 días, más ATT (6.000 U.I.) s.c. En el postoperatorio, aparecen: secreción nasal bilateral purulenta, tos, fiebre, linfocitosis y neutrofilia. El examen particular del sistema respiratorio (Dixon et al. 1995; Dixon 1997) incluyó: anamnesis, examen clínico general, auscultación y percusión de cavidad toráxica, rayos $\mathrm{X}$, hematología y bioquímica de rutina. En el examen radiológico del tórax, se observó un patrón bronquio-alveolar, sobretodo en el aspecto ventrocraneal de los pulmones; por lo cual, se le hicieron dos aspirados transtraqueales bajo técnica descrita en la literatura (Malikides et al. 2003; Hugues et al. 2008; Rossi et al. 2018), usando sedación con Xilacina i.v. (1 mg por kg p.v). Las muestras obtenidas se enviaron al Laboratorio de Bacteriología, Escuela de Medicina Veterinaria, Universidad Nacional, Costa Rica, para identificación del patógeno y pruebas de sensibilidad antibiótica (Caso No. EO35-18). El examen citológico del aspirado transtraqueal reveló gran cantidad de neutrófilos líticos. 
Rev. Ciencias Veterinarias, Vol. 38, N 1, [43-52], E-ISSN: 2215-4507, enero-junio, 2020

DOI: https://doi.org/10.15359/rcv.38-1.4

URL: http://www.revistas.una.ac.cr/index.php/veterinaria/index

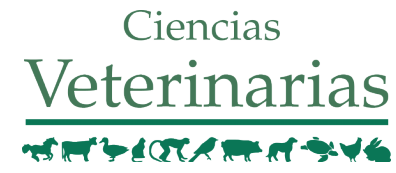

\section{Diagnóstico microbiológico}

El cultivo bacteriológico de una de las muestras obtenidas, por medio del aspirado transtraqueal, reveló resultados compatibles con Rodococcus equi.

\section{Aislamiento, identificación y prueba de susceptibilidad a los antibióticos (PSA).}

El aspirado transtraqueal se cultivó en Columbia agar $(\mathrm{BD})+5 \%$ sangre de carnero por 24 hs. a $37^{\circ} \mathrm{C}$. Las colonias aisladas fueron sometidas a tinción de Gram y a las siguientes pruebas fenotípicas y bioquímicas: CAMP, beta-hemolisis, reducción de nitrato, ureasa y fermentación de glucosa como descrito (Markley 2013). La prueba de susceptibilidad a los antibióticos se efectuó por el método de difusión en disco de Kirby Bauer sobre agar Mueller-Hinton usando seis antibióticos: ceftiofur (30 $\mu \mathrm{g})$, cefalotina $(30 \mu \mathrm{g})$, eritromicina $(15 \mu \mathrm{g})$, gentamicina $(10 \mu \mathrm{g})$, doxiciclina $(30 \mu \mathrm{g})$ y sulfametoxazole/trimetoprim $(25 \mu \mathrm{g})$ (OXOID) e interpretación de acuerdo al "Clinical and Laboratory Standards Institute" (CLSI 2015).

\section{Amplificación, secuenciación y determinación de 16S rRNA.}

Las bacterias se cultivaron en agar tripticasa soya; se incubaron por 1-2 días a $37^{\circ} \mathrm{C}$. Se suspendió una colonia individual en $50 \mu \mathrm{L}$ de agua PCR y se calentó a $95^{\circ} \mathrm{C}$ por $20 \mathrm{~min}$. La suspensión fue centrifugada a $10.000 \mathrm{rpm} / 5 \mathrm{~min}$. El sobrenadante se utilizó como plantilla de ADN. Se utilizó el GoTaq Green Master Mix (Promega) para montar la reacción de PCR, siguiendo las instrucciones del fabricante y $1.5 \mu \mathrm{L}$ de ADN y $0.4 \mu \mathrm{M}$ de los imprimadores 27F (5'-AGAGTTTGATCMTGGCTCAG-3') y 1492R (5'-ACCTTGTTACGACTT-3'). Las mezclas reaccionantes se incubaron $5 \mathrm{~min}$ a $95^{\circ} \mathrm{C}$. Luego, 35 ciclos se efectuaron como sigue: 30 s a $95^{\circ} \mathrm{C}, 30$ s a $55^{\circ} \mathrm{C}$ y 1 min 30 s a $72^{\circ} \mathrm{C}$. Con una extensión final de 10 min a $72^{\circ} \mathrm{C}$. Los productos del PCR se purificaron con QIAQuick Gel Extraction Kit (QIAGEN). La secuenciación se realizó en Macrogen (Macrogen Inc, South Korea), siguiendo metodologías establecidas; usando imprimadores $27 \mathrm{~F}$ and 1492R. Las secuencias genéticas parciales $16 \mathrm{~S}$ rRNA fueron alineadas usando MEGA7: Molecular Evolutionary Genetics Analysis Version X (Kumar et al. 2018). Después del alineamiento, la secuencia consensuada fue comparada con secuencias de nucleótido, hallados en BLAST (http://www.ncbi.nlm.nih.gov/BLAST/).

\section{Resultados}

\section{Aislamiento e identificación bioquímica}

Después de 24 hs. de incubación, se observó colonias lisas, brillantes y no hemolíticas. Estas colonias se hicieron más grandes, mucoides y color salmón, después de 48 hs. de incubación. La apariencia microscópica de la bacteria fue de bacilos y cocos pleomórficos Gram positivos, con predominio de formas cocoides. Las pruebas fenotípicas y bioquímicas resultantes fueron compatibles con $R$. equi (Cuadro 1). De los 6 antibióticos probados, la bacteria solo mostró perfil de resistencia hacia la cefalotina. Todos los otros antibióticos fueron susceptibles (Cuadro 2). 
Cuadro 1. Pruebas fenotípicas y bioquímicas

\begin{tabular}{ll} 
Prueba & Resultado \\
\hline CAMP & Incremento de hemolisina estafilocóccica \\
Beta-hemolisis & Negativa \\
Reducción de Nitratos & Positivo \\
Urease & Positiva $(>18 \mathrm{~h})$ \\
Fermentación glucosa & Negativa \\
\hline
\end{tabular}

Cuadro 2. Perfil de susceptibilidad a los antibióticos

\begin{tabular}{ll} 
Antibiótico & Interpretación \\
\hline Ceftiofur & Susceptible \\
Cefalotina & Resistente \\
Doxyciclina & Susceptible \\
Eritromicin & Susceptible \\
Gentamicina & Susceptible \\
Sulfametoxazole/Trimetoprim & Susceptible \\
\hline
\end{tabular}

\section{Secuenciación de 16S rRNA}

Los analisis BLAST, de la secuencia consensuada, mostraron cobertura del 98\% y $98 \%$ identidad a Rhodococcus sp. La definición de la especie requiere más análisis complementarios de ADN.

\section{Conclusiones e importancia clínica}

Es importante efectuar cultivo microbiológico y PSA en potrillos neumónicos (Giguère et al. 2010) para no incrementar la resistencia bacteriana con el uso de antibióticos inadecuados. El uso de liposomas, para transportar la variedad de moléculas usadas en investigación biomédica y práctica clínica, ha demostrado su eficiencia (Alhariri et al. 2013) pues mejoran el índice terapéutico de los agentes encapsulados y reducen la toxicidad de la droga, al mostrar, estos "antibióticos liposomales con entrega dirigida", un interesante potencial para tratamiento de infecciones microbianas. La gentamicina liposomal intravenosa puede ser efectiva para el tratamiento de neumonía por R. equi; sin embargo, la nefrotoxicidad, encontrada con esta vía, indica que un protocolo alternativo (como nebulización) es necesario para el uso clínico seguro del medicamento (Cohen et al. 2016). Otras estrategias terapéuticas reportan resultados variados, tales como: plasma hiperinmune o inmunoglobulinas (HooperMcGrevy et al. 2001); Interferón-Gamma (Sturgill \& Horohov 2006); Maltolato de Galio (Chaffin et al. 2011; Cohen et al. 2015) y algunas otras nanoformulaciones (Sharma et al. 2012; Singh et al. 2014; Nachimuthu et al. 2017; Baptista et al. 2018); pero se requieren más estudios clínicos comparativos para evaluar su eficacia y seguridad relativa. El potencial profiláctico de la vacuna, usando anticuerpos 
a la PNAG (Cywes-Bentley et al. 2018), ha generado grandes expectativas en la industria equina, pues provee protección a los potrillos contra varios patógenos intracelulares, incluyendo $R$. equi. Con este protocolo, las yeguas madres son vacunadas 6 a 3 semanas antes de la posible fecha de parto, siendo los anticuerpos transferidos a los potrillos exclusivamente vía calostro.

\section{Recomendaciones}

- Recoger heces del ambiente y eliminarlas adecuadamente, sin permitir que se pulvericen, para prevenir contagio por inhalación.

- Supeditado al monitoreo clínico del paciente, se debe reconsiderar el uso de antibióticos en animales con lesiones pulmonares subclínicas y ultrasonográficamente pequeñas, pues según reportes la mayoría de estos casos se recuperan sin terapia.

- Prescribir antibióticos basados, solamente, en resultados de cultivo y PSA.

- Promover programas regulares de inmunización apenas esté disponible una vacuna comercial registrada.

\section{Referencias}

Al Akhrass, F., Al Wohoush, I., Chaftari, A.M., Reitzel, R., Jiang, Y., Ghannoum, M., Tarrand, J., Hachem, R. \& Raad, I. 2012. Rhodococcus bacteremia in cancer patients is mostly catheter related and associated with biofilm formation. PLOS ONE 7(3): e32945. doi: 10.1371/journal. pone. 0032945

Alhariri, M., Azghani, A. \& Omri, A. 2013. Liposomal antibiotics for the treatment of infectious diseases. Expert Opin. Drug Del. 10(11): 1515-1532. doi: 10.1517/17425247.2013.822860

Álvarez, M., Güiris, D.M., Samayoa, Y.Y., Cruz, E. \& Lira, I. 2001. Identification of aerobic bacteria from internal organs of Tapirus bairdii. Proceedings from First International Tapir Symposium, San José, Costa Rica, November 3-8, 2001. Abstract number 32. Instituto de Historia Natural y Ecología del Estado de Chiapas (IHNE) Apdo. Postal N6, C.P. 29000, Tuxtla Gutiérrez, Chiapas, México.

Baptista, P.V., McCusker, M.P., Carvalho, A., Ferreira, D.A., Mohan, N.M., Martins, M. \& Fernandes, A.R. 2018. Nano-Strategies to fight multidrug resistant bacteria - "A battle of the titans". Front. Microbiol. 9: 1441. doi: 10.3389/fmicb.2018.01441

Chaffin, M.K., Cohen, N.D., Martens, R.J., O’Connor, M. \& Bernstein, R. 2011. Evaluation of the efficacy of gallium maltolate for chemoprophylaxis against pneumonia caused by Rhodococcus equi infection in foals. Am. J. Vet. Res. 72(7): 945-957. doi: 10.2460/ajvr.72.7.945

CLSI. 2015. M02-A12 Performance Standards for Antimicrobial Disk Susceptibility Tests; Approved Standard - Twelfth Edition, $12^{\text {th }}$ Edition. Clinical and Laboratory Standards Institute, Wayne, Pennsylvania. 
Cohen, N.D., Carter, C.N., Scott, M., Chaffin, K., Smith, J.L., Grimm, M.B., Kuskie, K.R., Takai, S. \& Martens, R.J. 2008. Association of soil concentrations of Rhodococcus equi and incidence of pneumonia attributable to Rhodococcus equi in farms of central Kentucky. Am. J. Vet. Res. 69 (3): 385-395. doi: 10.2460/ajvr.69.3.385

Cohen N.D., Kuskie, K.R., Smith, J.L., Slovis, N.M., Brown, S.E., Stepusin, R.S., Chaffin, M.K., Takai, S. \& Carter, C.N. 2012. Association of airborne concentration of virulent Rhodococcus equi with location (stall versus paddock) and month (January through June) on 30 horse breeding farms in central Kentucky. Am. J. Vet. Res. 73(10): 1603-1609. doi: 10.2460/ajvr.73.10.1603

Cohen N.D., Chaffin, M.K., Kuskie, K.R., Syndergaard, M.K., Blodgett, G.P. \& Takai, S. 2013. Association of perinatal exposure to airborne Rhodococcus equi with risk of pneumonia caused by $R$. equi in foals. Am. J. Vet. Res. 74(1): 102-109. doi: 10.2460/ajvr.73.10.160

Cohen, N.D., Slovis, N.M., Giguére, S., Baker, S., Chaffin, M.K. \& Bernstein, L.R. 2015. Gallium maltolate as an alternative to macrolides for treatment of presumed Rhodococcus equi pneumonia in foals. J. Vet. Intern. Med. 29(3): 932-939. doi: 10.1111/jvim.12595

Cohen, N.D., Giguère, S., Burton, A.J., Rocha, J.N. Berghaus, L.J., Brake, C.N., Bordin, A.I. \& Coleman, M.C. 2016. Use of liposomal gentamicin for treatment of 5 foals with experimentally induced Rhodococcus equi pneumonia. J. Vet. Intern. Med. 30(1): 322-325. doi: 10.1111/jvim.13810.

Cywes-Bentley, C., Rocha, J.N., Bordin, A.I., Vinacur, M., Rehman, S., Zaidi, T.S., Meyer, M., Anthony, S., Lambert, M., Vlock, D.R., Giguère, S., Cohen, N.D. \& Pier, G.B. 2018. Antibody to Poly- $N$-acetyl glucosamine provides protection against intracellular pathogens: Mechanism of action and validation in horse foals challenged with Rhodococcus equi. Plos Pathog. 14(7): e1007160. doi: 10.1371/journal.ppat.1007160

Dixon, P.M. 1997. Ancillary diagnostic techniques for the investigation of equine pulmonary disease. Equine Vet. Educ. 9(2): 72-80. doi: 10.1111/j.2042-3292.1997.tb01281.x

Dixon, P.M., Railton, D.I. \& McGorum, B.C. 1995. Equine pulmonary disease: a case control study of 300 referred cases. Part 1: Examination techniques, diagnostic criteria and diagnosis. Equine Vet. J. 27(6): 416-421 https://doi.org/10.1111/j.2042-3306.1995.tb04421.x

Forbes, B., Sahm, D. \& Weissfeld, A. 1998. Nocardia, Streptomyces, Rhodococcus, Oerskovia and similar organisms. Bailey \& Scott's Diagnostic Microbiology, 10 ${ }^{\text {th }}$ Edition. Mosby, Estados Unidos.

Giguère, S., Lee, E., Williams, E., Cohen, N.D., Chaffin, M.K., Halbert, N., Martens, R.J., Franklin, R.P., Clarck, C.C. \& Slovis, N.M. 2010. Determination of the prevalence of antimicrobial resistance to macrolide antimicrobials or rifampin in Rhodococcus equi isolates and treatment outcome in foals infected with antimicrobial-resistant isolates of $R$ equi. JAVMA-J. Am. Vet. Med. A. 237(1): 74-81. doi: 10.2460/javma.237.1.74

Guiguère, S. 2010. Infecciones por Rhodococcus equi. En: Smith, B.P. (Ed.). Medicina Interna de Grandes Animales. Elsevier España S.L, Barcelona, p. 510-520.

Giguère, S. 2017. Treatment of infections caused by Rhodococcus equi. Vet. Clin. North Am. Equine Pract. 33(1): 67-85. doi: 10.1016/j.cveq.2016.11.002 
Rev. Ciencias Veterinarias, Vol. 38, N 1, [43-52], E-ISSN: 2215-4507, enero-junio, 2020

DOI: https://doi.org/10.15359/rcv.38-1.4

Ciencias

URL: http://www.revistas.una.ac.cr/index.php/veterinaria/index

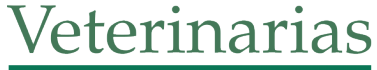

Gressler, L.T., de Vargas, A.C., da Costa, M.M., Sutili, F.J., Schwab, M., Pereira, D.I., Sangioni, L.A. \& Botton, S. 2015. Biofilm formation by Rhodococcus equi and putative association with macrolide resistance. Pesq. Vet. Bras. 35(10): 835-841. doi: 10.1590/S0100-736X2015001000003

Grimm, M.B., Cohen, N.D., Slovis, N.M., Mundy, G.D., Harrington, J.R., Libal, M.C., Takai, S. \& Martens, R.J. 2007. Evaluation of fecal samples from mares as a source of Rhodococcus equi for their foals by use of quantitative bacteriologic culture and colony immunoblot analyses. Am. J. Vet. Res. 68(1): 63-71. doi: 10.2460/ajvr.68.1.63

Herrera, M.L., Vargas, A. \& Campos, M. 2000. Primer aislamiento clínico de Rhodococcus equi en Costa Rica. Rev. Méd. Hosp. Nac. Niños (Costa Rica) 35(1-2): 49-53.

Hooper-McGrevy, K.E., Giguere, S., Wilkie, B.N. \& Prescott, J.F. 2001. Evaluation of equine immunoglobulin specific for Rhodococcus equi virulence-associated proteins $\mathrm{A}$ and $\mathrm{C}$ for use in protecting foals against Rhodococcus equi-induced pneumonia. Am. J. Vet. Res. 62(8): 1307-1313. doi: 10.2460/ajvr.2001.62.1307

Hugues, K.J., Malikides, S.N., Hodgson, D.R. \& Hodgson, J.L. 2008. Comparison of tracheal aspirates and bronchoalveolar lavage in racehorses 1. Evaluation of cytological stains and the percentage of mast cells and eosinophils. Aust. Vet. J. 81(11): 681-684. doi: 10.1111/j.1751-0813.2003.tb12538.x

Kelly, B.G, Wall, D.M., Boland, C.A. \& Meijer, W.G. 2002. Isocitrate lyase of the facultative intracellular pathogen Rhodococcus equi. Microbiology 148(3): 793-798. doi: 10.1099/00221287-148-3-793.

Kumar, S., Stecher, G., Li, M., Knyaz, C. \& Tamura, K. 2018. MEGA X: Molecular evolutionary genetics analysis across computing platforms. Mol. Biol. Evol. 35(6): 1547-1549. doi: 10.1093/molbev/msy096

Kuskie, K.R., Smith, J.L., Wang, N., Carter, C.N., Chaffin, M.K., Slovis, N.M., Stepusin, R.S., Cattoi, A.E., Takai, S. \& Cohen, N.D. 2011. Effects of location for collection of air samples on a farm and time of day of sample collection on airborne concentrations of virulent Rhodococcus equi at two horse breeding farms. Am. J. Vet. Res. 72(1): 73-79. doi: 10.2460/ajvr.72.1.73

Lasky, J., Pulkingham, N., Powers, M.A. \& Durack, D.T.1991. Rhodococcus equi causing human pulmonary infection: review of 29 cases. South Med. J. 84(10): 1217-1220.

Letek, M., Ocampo-Sosa, A.A., Sanders, M., Fogarty, U., Buckley, T., Leadon, D.P., González, P., Scortti, M., Meijer, W.G., Parkhill, J., Bentley, S. \& Vázquez-Boland, J.A. 2008. Evolution of the Rhodococcus equi vap pathogenicity island seen through comparison of host-associated vapA and vapB virulence plasmids. J. Bacteriol. 190(17): 5797-5805. doi: 10.1128/JB.00468-08

Losinger, W.C., Traub-Dargatz, J.L., Sampath, R.K. \& Morley, P.S. 2000. Operation-management factors associated with early-postnatal mortality of US foals. Prev. Vet. Med. 47(3): 157-175. doi: 10.1016/ S0167-5877(00)00173-2

Malikides, S.N., Hughes, K.J., Hodgson, D.R. \& Hodgson, J.L. 2003. Comparison of tracheal aspirates and bronchoalveolar lavage in racehorses 2 . Evaluation of the diagnostic significance of neutrophil percentage. Aust. Vet. J. 81(11): 685-687. doi: 10.1111/j.1751-0813.2003. tb12540.x.

Nachimuthu, N., Manohar, P., Shanthini, T., Gothandam, K.M., Karthikeyan, S. \& Bulent, B. 2017. 


\section{Ciencias}

Nano-antibiotics: A Therapeutic Future. Nanoscience and Nanotechnology - Asia. 7(1): 3-25. doi: $\underline{10.2174 / 2210681207666170215151010}$

Markey, B., Leonard, F., Archambault, M. \& Cullinane, A. 2013. Clinical Veterinary Microbiology, 2nd Edition. Elsevier, Estados Unidos. 920p.

Muscatello, G, Leadon, D.P., Klayt, M., Ocampo-Sosa, A., Lewis, D.A., Fogarty, U., Buckley, T., Gilkerson, J.R., Meijer, W.G. \& Vazquez-Boland, J.A. 2007. Rhodococcus equi infection in foals: the science of 'rattles'. Equine Vet. J. 39(5): 470-478. doi: 10.2746/042516407X209217

Prescott, J.F. 1987. Epidemiology of Rhodococcus equi infection in horses. Vet. Microbiol. 14(3): 211-214. doi: $10.1016 / 0378-1135(87) 90107-6$

Prescott, L., Harley, J. \& Klein, D. 1997. The Bacteria: The Actinomycetes. Microbiology, $2^{\text {nd }}$ Edition. McGraw-Hill Education, Estados Unidos.

Rossi, H., Virtala, A.M., Raekallio, M., Rahkonen, E., Rajamaki, M.M. \& Mykkanen, A. 2018. Comparison of tracheal wash and bronchoalveolar lavage cytology in 154 horses with and without respiratory signs in a referral hospital over 2009-2015. Front. Vet. Sci. 5(61). doi: 10.3389/fvets.2018.00061

Sharma, A., Kumar, D., Dua, M., Chhatwal, G.S. \& Johri, A.K. 2012. Nano-technologies for targeted drug delivery to combat antibiotic resistance. Expert Opin. Drug Del. 9(11): 1325-1332. doi: $\underline{10.1517 / 17425247.2012 .717927}$

Singh, R., Smitha, M.S. \& Singh, R. 2014. The role of nanotechnology in combating multi-drug resistant bacteria. J. Nanosci. Nanotechnol. 14(7): 4745-4756. doi: 10.1166/jnn.2014.9527

Sturgill, T.L. \& Horohov, D.W. 2006. Interferon-Gamma expression in young foals when treated with an immunostimulant or plasma. P. Annu. Conv. Am. Equin. 52: 237-241.

Takai, S, Hines, S.A., Sekizaki, T., Nicholson, V.M., Alperin, D.A., Osaki, M., Takamatsu, D., Nakamura, M., Suzuki, K., Ogino, N., Kakuda, T., Dan, H. \& Prescott, J.F. 2000. DNA sequence and comparison of virulence plasmids from Rhodococcus equi ATCC 33701 and 103. Infect. Immun. 68(12): 6840-6847. doi: 10.1128/iai.68.12.6840-6847.2000

Zachary, J.F. 2017. Pathological basis of Veterinary Disease, $6^{\text {th }}$ Edition. Elsevier, St. Louis, Estados Unidos. 526p. 